\title{
TЕХНИЧЕСКИЕ НАУКИ
}

\section{ОЦЕНКА ВЛИЯНИЯ ИСКУССТВЕННОГО ИНТЕЛЛЕКТА НА ОПЕРАТИВНОЕ УПРАВЛЕНИЕ УЧАСТКОВОЙ ЖЕЛЕЗНОДОРОЖНОЙ СТАНЦИИ}

\author{
Бельх Алексей Алексеевич \\ студент 5 курса \\ направление «Эксплуатация железных дорог», \\ Дальневосточный Государственный Университет Путей Сообщения \\ 2. Хабаровск \\ Широкова Валентина Васильевна, \\ Кандидат педагогических наук, доиент кафедры \\ «Организация перевозок и безопасность на транспорте», \\ Дальневосточный Государственный Университет Путей Сообщения, \\ г.Хабаровск
}

\section{ASSESSMENT OF THE IMPACT OF ARTIFICIAL INTELLIGENCE ON THE OPERATIONAL MANAGEMENT OF THE PRECINCT RAILWAY STATION}

Belykh Alexey

Fifth year student «railway operation », Far Eastern State Transport University, Khabarovsk Shirokova Valentina, Candidate of Pedagogic Sciences Far Eastern State Transport University, Khabarovsk DOI: $10.31618 /$ nas.2413-5291.2020.1.56.229

\section{Аннотация}

Данная статья посвящена решению задачи в области оперативного управления железнодорожной станцией $\mathrm{c}$ использованием нейронных сетей. В статье сформулированы основные принципы программного обеспечения и рассмотрена его архитектура. Предложен технологический график работы дежурного по станции с применением искусственного интеллекта. Выполнена оценка воздействия искусственного интеллекта на оперативное управление в работе диспетчерского аппарата.

\section{Abstract}

This article dedicated to solving the problem in the field of operational management of the railway station using neural networks. The article outlines the basic principles of the software and examines its architecture. The technological schedule of the station attendant with the use of artificial intelligence proposed. An assessment of the impact of artificial intelligence on operational control in the operation of the control room carried out.

Ключевые слова: нейронные сети; железнодорожная станция.

Keywords: neural network; railway station.

На участковой станции принятие решение возлагается на дежурных по станции(ДСП), и руководителя смены - маневрового диспетчера. Дежурные по станции обеспечивают, регулируют, контролируют движение поездов на станции, в частности их прибытие, отправление, проследование. В оперативной работе они руководствуются приказами диспетчера поездного. С учетом оперативно складывающейся обстановки вся работа организуется в условиях полного обеспечения безопасности, а также выполнения сменно-суточных показатели.

С этой целью необходимо в режиме реального времени получать информацию о текущей обстановке на станции, прогнозировать всевозможные варианты развития событий и принимать оптимальное решение по организации движения.

В свою очередь повышение интенсивности и скоростей следования поездов требуют совершенствования систем железнодорожной автоматики в частотности, систем управления движения поездов и систем безопасности и управления на подвижном составе, увеличения информативности, снижения влияния человеческого фактора, повышения уровня безопасности движения.

За короткое время, которое имеется для принятия оперативных управляющих решений (ОУР), человеку-диспетчеру невозможно просчитать эффективность различных решений, учесть большое число влияющих на перевозочный процесс параметров. Поэтому опыт и интуиция оперативного персонала сегодня играют решающую роль в принятии ОУР.

Переход к интеллектуальным автоматизированным рабочим местам(АРМ) состоит в том, что выработка ОУР осуществляется по специальным алгоритмам на основе разработанной имитационной модели работы управляемой станции с использованием необходимого комплекса данных информационноуправляющих систем. При этом должно учитываться максимальное число влияющих 
параметров и опыт лучших оперативных работников. Также должна обязательно реализовываться функция самообучения системы [3].

Для каждой группы поездов и вагонов данной структуры необходимо учитывать свою специфику прогнозирования их подхода. Если оперативные процессы проходят в рамках существующей технологии, то диспетчерский персонал выполняет лишь функцию контроля хода перевозочного процесса. Например, если все поезда следуют строго по графику, то каких-либо оперативных управляющих решений принимать не требуется. В случае отклонения от действующей технологии необходимо принятие ОУР.

В настоящий момент системы прогнозирования основаны на такой технологии работы и принятии решений, в которой логика строго регламентирована и отступлений быть не может. В связи с этим моделирование текущих процессов на станции строго ограничено и возможность их оптимизации мало осуществимы.

Таким образом, моделирование движения на железнодорожном транспорте должно быть таким, и преследовать такую логику, в которой порядок принятия решений будет сформулирован уже заранее. При этом появляется возможность вносить изменения в систему с минимальными трудозатратами и финансовыми капиталовложениями, так как процесс перевозок это динамичный процесс.

Для достижения поставленных целей предлагается решить ряд задач:

1. Автоматизировать процесс принятия решений по пропуску грузовых и пассажирских поездов по отдельным элементам станции в различных режимах работы.

2. Разработать и повсеместно внедрять интеллектуальные программные комплексы, в которых реализована возможность совершенствования отдельных элементов комплекса в процессе создания и непосредственной эксплуатации.

Особый интерес представляет метод решения данной задачи с помощью нейросетевых технологий или искусственных нейронных сетей (ИНС). ИНС представляют собой вычислительные структуры, которые моделируют биологические процессы, обычно ассоциируемые с процессами человеческого мозга. Они способны решать широкий круг задач прогнозирования, оптимизации, управления сложными организационно-техническими системами. ИНС позволяют решать не формализуемые или трудно формализуемые задачи. Очень важно, что ИНС способны к адаптивному обучению по экспериментальным данным (опыту специалистов) без построения моделей, поэтому их использование особенно эффективно для сложных систем, когда математическая модель или отсутствует, или неоправданно сложна [4].

Основные преимущества нейросетевых технологий:

1. Возможность учесть не формализуемые зависимости (за счет обучения на примерах).

2. С помощью нейросетей можно выявлять скрытые зависимости через весовые коэффициенты. Выявлять наиболее значимые зависимости.

3. Возможность решения задачи многофакторной оптимизации (решение обратной задачи при известном min на выходе).

Одним из важнейших требований, которые предъявляются к модели, ее универсальность и модульность. Поскольку, одной из основных задач, которые предъявляются к системе, это принятие решений. Эти решения будут касаться оценки различных вариантов организационных мероприятий, осуществляемых на станции.

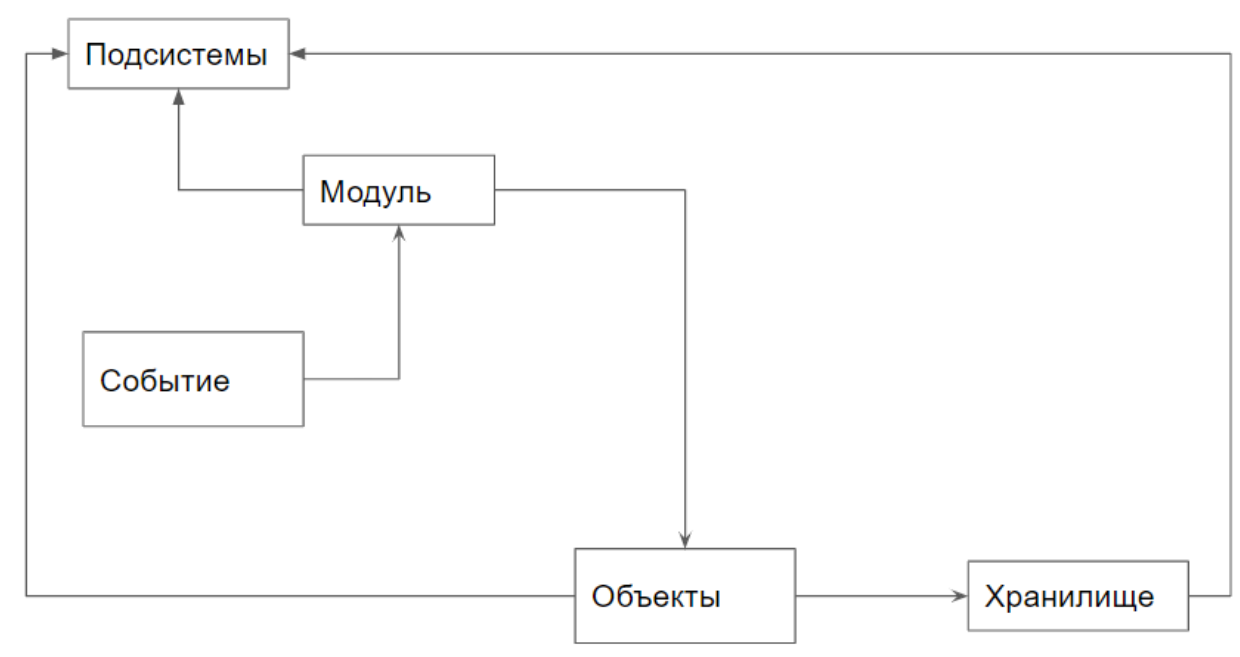

Рисунок 1. Диаграмма базовых компонентов

Модульная организация позволяет легко модифицировать итоговую модель в соответствии с характером решаемой задачи, а также упрощает синтез моделей для конкретных станций, позволяя учесть особенности их технического оснащения и технологии работы. При этом одним из основных 
требований к отдельным модулям является простота и универсальность представления исходных данных за счет усложнения внутренней организации моделирующих программных комплексов.
Концепция проекта представленная на рисунке 2, основывается на построении бесшовной интеграции с существующими подсистемами и возможностью сохранения стабильности и целостности межмодульных интерфейсов.

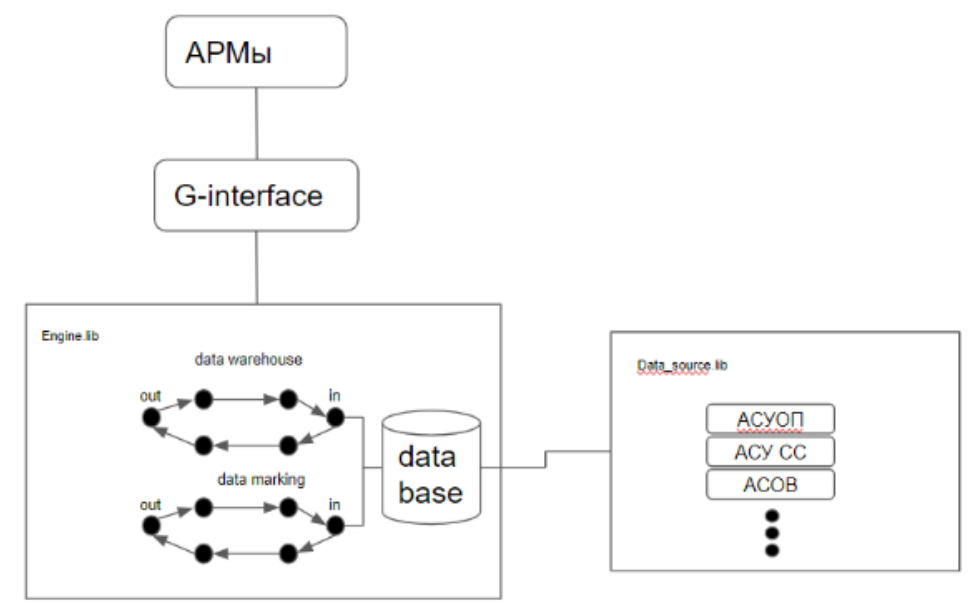

Рисунок 2. Общая концฺепцุия потока данных в системе

Онтология строится вокруг модулей, которые являются центральной частью модели. Каждый модуль рассмотрен и спроектирован с учетом требований затрат минимальных ресурсов для обеспечения бесперебойной работы. Важно, чтобы модель была достоверной и полной и модули связаны между собой [1].
В задачах машинного обучения качество моделей сильно зависит от данных. И в реальных задачах, чаще всего редко бывают идеальными. Как правило это связанно, с анализом параметров, шумом, и пропусками.

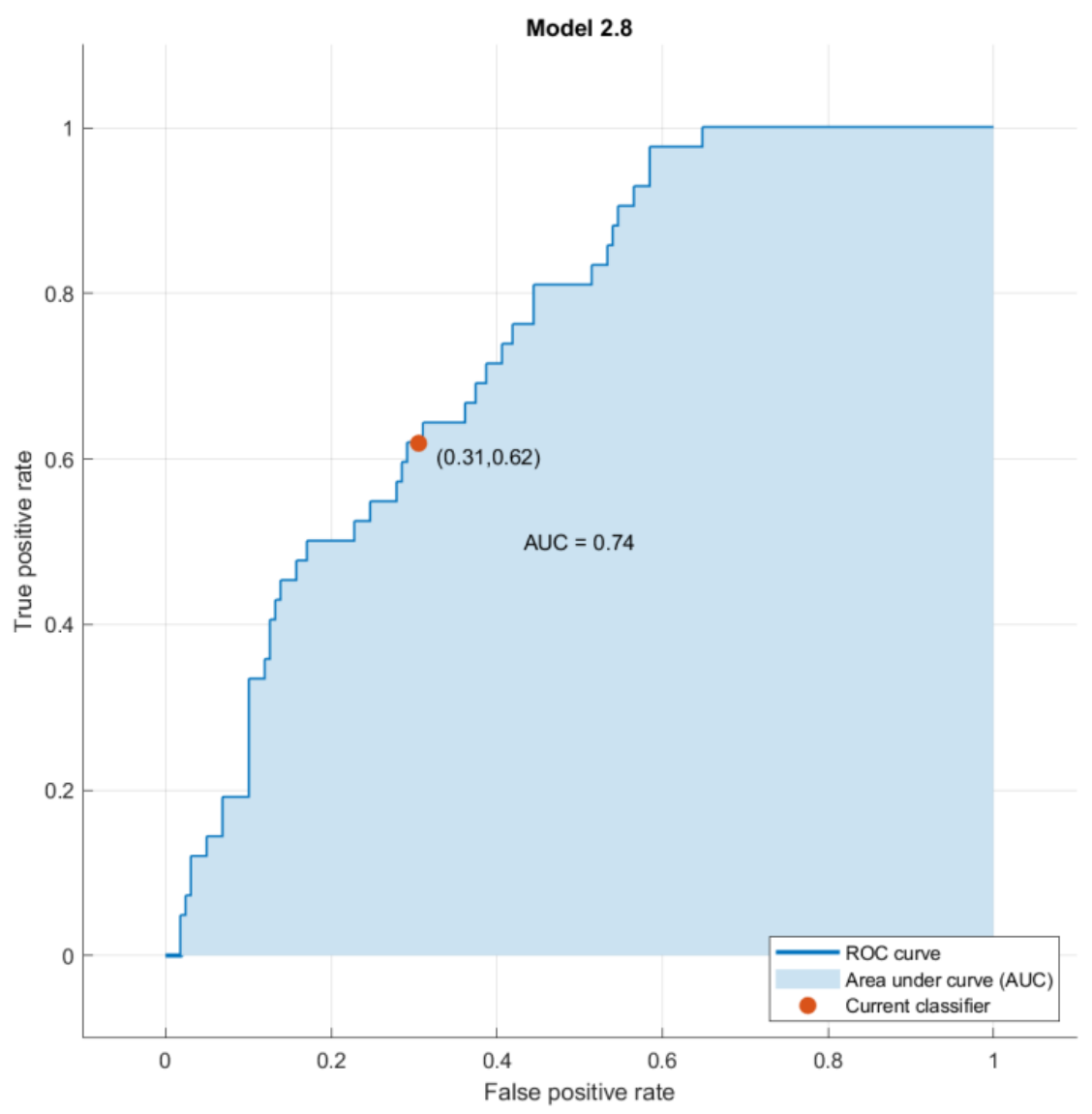

Рисунок 3. ROC - кривая для метода опорных векторов 
Так, на рисунке 4 видно, что процент ложных срабатываний равный 0,31 указывает на то, что текущий классификатор неправильно присваивает $31 \%$ наблюдений положительному классу. Истинный положительный коэффициент 0,61 указывает, что текущий классификатор правильно присваивает $61 \%$ наблюдений положительному классу.

В ходе проведения экспериментов и выбора структуры нейронной сети, установлено, что для решения задачи прогнозирования пути приема поездов следует использовать нейронную сеть, построенную по методу прогнозирования NARX.

Эффективность модели, построенной на основе нейронной сети, в составе единой интеллектуальной системы управления станцией определяется исходя из повышения эффективности производительности труда оперативнодиспетчерского персонала [2]. В частности, модель прогнозирования выбора пути приема поезда позволяет уменьшить нагрузку на дежурного по станции; дает возможность минимизации дублирования операций без ухудшения результатов процесса, а также экономию трудоемкости выполнения операций. На рисунке 4, продемонстрирована диаграмма сравнения до и после внедрения автоматизации в оперативное управление железнодорожной станции.

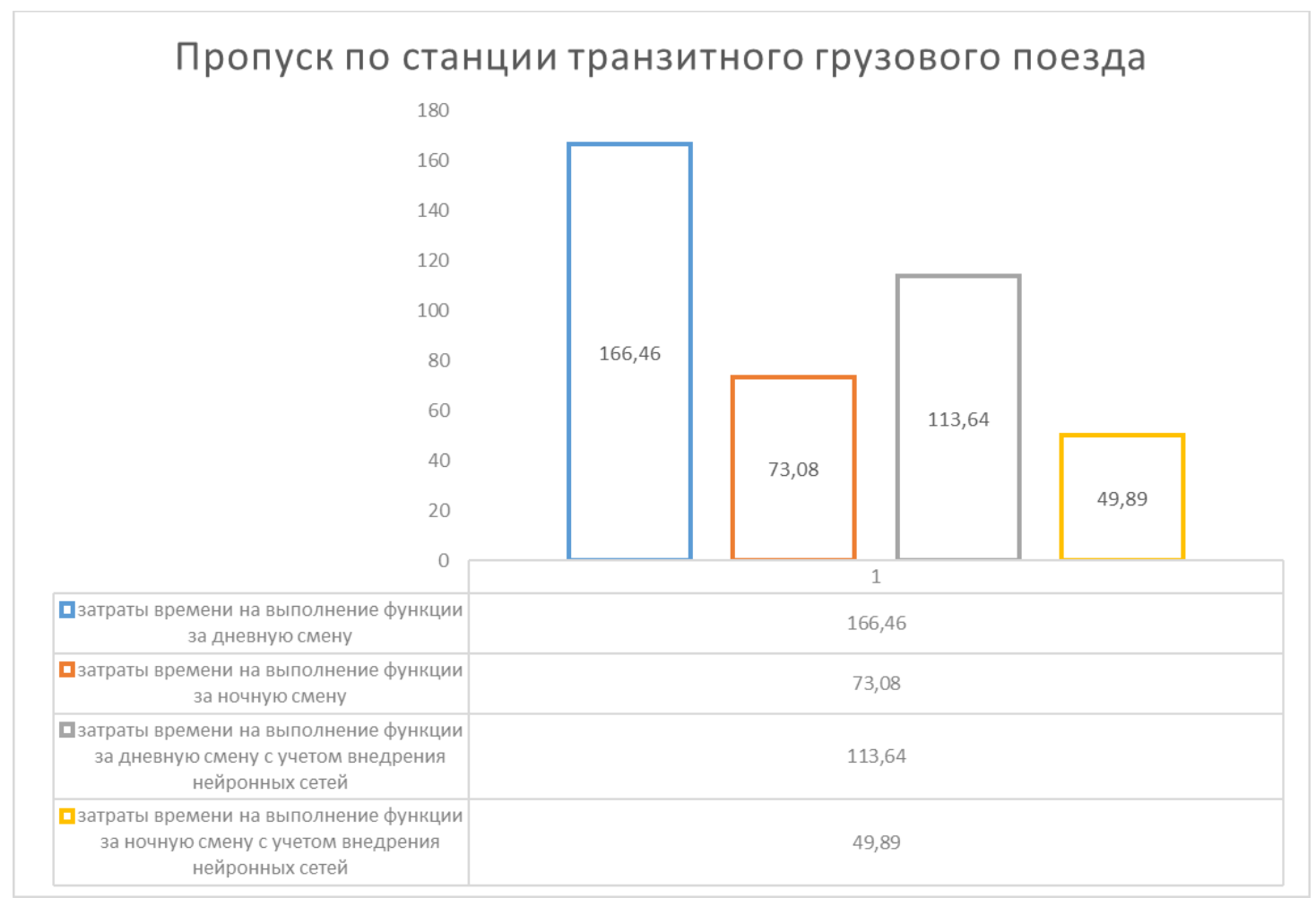

Рисунок 4. Диаграмма сравнения трудозатрат ДСП участковой станиฺии

Нейросетевая модель оперативного управления позволяет наращивать мощности участковой станции. При этом наибольший эффект может быть достигнут на основе совершенствования методов управления по всему комплексу станционных технологических процессов и операций. 


\begin{tabular}{|c|c|c|c|}
\hline $\begin{array}{r}\text { начало счета: } \\
\text { масштаб счета }\end{array}$ & & & \\
\hline Операторы и логические условия алгоритма & $\begin{array}{l}\text { начало } \\
\text { операции }\end{array}$ & $\begin{array}{l}\text { конец } \\
\text { операции }\end{array}$ & $\begin{array}{l}\text { Затраты рабочего времени } \\
\text { на выполнение операций } \\
\text { алгоритма }\end{array}$ \\
\hline S0501 - считывание с часов фактического времени; & 0,00 & 12,00 & 12 \\
\hline S0502 - считывание с системы ГИД-Урал времени прибытия поезда; & 12,00 & 21,00 & 9 \\
\hline $\begin{array}{l}\text { S0503 - считывание информации с табло (монитора) о наличии свободного } \\
\text { пути приема; }\end{array}$ & 21,00 & 39,00 & 18 \\
\hline D0502 - переговоры с поездным диспетчером; & 39,00 & 79,00 & 40 \\
\hline $\begin{array}{l}\text { А0501 - принятие решения об остановке поезда у входного светофора } \\
\text { станции; }\end{array}$ & 79,00 & 85,00 & 6 \\
\hline $\begin{array}{l}\text { А0502 - контроль проследования поезда с помощью графической } \\
\text { визуализации }\end{array}$ & 85,00 & 89,00 & 4 \\
\hline $\begin{array}{l}\text { R0501 - запись в журнал (ДУ-3) время проследования транзитного грузового } \\
\text { поезда по станции; }\end{array}$ & 89,00 & 113,00 & 24 \\
\hline & \multicolumn{2}{|c|}{ Итого } & 113 \\
\hline \multicolumn{4}{|l|}{ Логические условия } \\
\hline \multicolumn{4}{|l|}{$\begin{array}{l}\text { Считывание информации с часов, пульт-табло, мониторов АСУ, журналов и } \\
\text { рабочих записей }\end{array}$} \\
\hline \multicolumn{4}{|l|}{ Использование Ии } \\
\hline Запись информации в журналы и АСУ & & & \\
\hline
\end{tabular}

Рисунок 5. Технологический график операторов и логических условий функции пропуска по станциии транзитного грузового поезда без изменения массы и длины/смены локомотива или локомотивной бригады на участковой железнодорожной станции с применением искусственного интеллекта в дневную смену

\begin{tabular}{|c|c|c|c|}
\hline \multirow[b]{2}{*}{ Oператоры и погические усповия алгоритми } & \multicolumn{2}{|c|}{$\begin{array}{l}1,00 \\
8,4\end{array}$} & \multirow[b]{2}{*}{$\begin{array}{l}\text { Затраты рабочего времени на } \\
\text { выполнение операций } \\
\text { алгоритма, мин }\end{array}$} \\
\hline & $\begin{array}{l}\text { мачвло } \\
\text { опервции }\end{array}$ & $\begin{array}{l}\text { конец } \\
\text { операции }\end{array}$ & \\
\hline S0501 - сиитаивание с иасов фактического времети; & 0,00 & 12,00 & 12 \\
\hline 50502 - сиитавание с системы ПИД-Урал времети прибытия поезда; & 12,00 & 21,00 & 9 \\
\hline $\begin{array}{l}\text { S0503 - считывание информации с табло (монитора) О маличии сзободного пути } \\
\text { приема; }\end{array}$ & 21,00 & 39,00 & 18 \\
\hline A0501 - вы6ор пути пропуска; & 39,00 & 48,00 & 9 \\
\hline Р0501 - свободамый путь Аля пропуска поезда отсүтствует, & 0,00 & 0,00 & \\
\hline В0501 - переход алгоритма к операторам при наличии сзободмого пути: & 0,00 & & \\
\hline D0502 - переговоры с поеsаным диспетчером; & 48,00 & 88,00 & 40 \\
\hline А0501 - принятие решения о6 остановке поезда у входного светофора станции; & 88,00 & 94,00 & 6 \\
\hline $\begin{array}{l}\text { (К0504 - количество повторных считьіваний имформации с табло (монитора) об } \\
\text { освобождении пути приема поезда; }\end{array}$ & 94,00 & 103,50 & 9,5 \\
\hline $\begin{array}{l}\text { 50504) - сиитывание информации с та6ло (монитора) о6 освобождении пути приема } \\
\text { поезда; }\end{array}$ & 103,50 & 113,00 & 9,5 \\
\hline [; & 113,00 & 135,00 & 22 \\
\hline $\begin{array}{l}\text { Ш|К0505 - количество повторных считыізаний информации с та6ло о проследовании } \\
\text { траняитного груэового поезда по статции; }\end{array}$ & 135,00 & 138,50 & 3,5 \\
\hline S0505) - комтроль ислольsоsamия маршрута по та6ло (мотитору): & 138,50 & 142,00 & 3,5 \\
\hline \multirow[t]{2}{*}{$\begin{array}{l}\text { R0501 - sanись в турнал (ДУ-3) время проследования траняиттого грузоsoro поеsда по } \\
\text { станции; }\end{array}$} & 142,00 & 166,00 & 24 \\
\hline & \multicolumn{2}{|c|}{ Итoro } & 166 \\
\hline \multicolumn{4}{|l|}{ Условные обозначения операторов и логических функций } \\
\hline \multicolumn{4}{|l|}{ Логические условия } \\
\hline \multicolumn{4}{|l|}{$\begin{array}{l}\text { Сиитывание информации с часов, пульттабло, мониторов АCУ, журталоs и рабочих } \\
\text { записей }\end{array}$} \\
\hline \multicolumn{4}{|l|}{ Испольsование Ии } \\
\hline \multicolumn{4}{|l|}{ Запись информации в журталы и АCY } \\
\hline \multicolumn{4}{|l|}{ 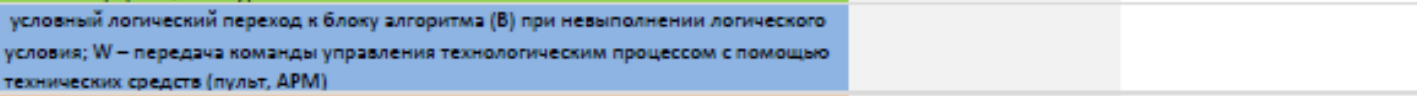 } \\
\hline К - количество повторений 6 пока алгоритиа, заключенного s скобки & & & \\
\hline
\end{tabular}

Рисунок 6. Технологический график операторов и логических условий функции пропуска по станциии транзитного грузового поезда без изменения массы и длины/смены локомотива или локомотивной бригады на участковой железнодорожной станции до применения искусственного интеллекта 8 дневную смену 
На рисунке 5 представлен алгоритм действий в обстановке с применением разработанных модулей, построенных на нейронной сети. Рисунок 6 в свою очередь представляет алгоритм действий дежурного по станции до внедрения автоматизации в рабочий процесс станции.

На основе результатов, полученных в данной работе, можно заключить что возможность применения искусственного интеллекта в работе диспетчерского аппарата в частотности, прогнозирования выбора пути приема возможна, поскольку наблюдается существенное повышение производительности труда диспетчерского аппарата.

\section{Список литературы:}

1. Клепов, А.В. Онтология ИСУЖТ [Текст]/ А.В. Клепов, В.А. Броневицкий, Н.И. Капустин // Труды седьмой научно - технической конференции интеллектуальные системы управления на железнодорожном транспорте. Компьютерное и математическое моделирование (ИСУЖТ-2018)/ АО «НИИАС» - Москва,2018. - С. 16-20.

2. Ковалев, К.Е. Метод распределения функций и зон управления между оперативным персоналом крупных участковых станций [Текст]: дис. ... канд. тех. наук: 05.22.08: защищена 15.12.2015 / Ковалев Константин Евгеньевич. СПб., 2015. - 194 с. - 416012680024

3. Обухов А.Д. Нейронные сети: распознавание, управление, принятие решений [Текст]: дис. ... канд. тех. наук: 05.22.08.: защищена 30.05.2017 / Обухов Андрей Дмитриевич. - СПб., 2016. - 146 c. - AАAА-B17-417061550044-1

4. Сотников Е.А., Интеллектуализация оперативного управления перевозочным процессом на уровне региональной дирекции управления движением [Текст] / Сотников Е.А. // Труды третьей научно - технической конференции интеллектуальные системы управления на железнодорожном транспорте. Компьютерное и математическое моделирование (ИСУЖТ-2014)/ АО «НИИАС» - Москва,2014. - С. 94-95.

\title{
ВОССТАНОВЛЕНИЕ АВТОМОБИЛЬНЫХ ДОРОГ ПОСЛЕ РЕМОНТА КОММУНИКАЦИЙ
}

\author{
Дормидонтова Татьяна Владимировна \\ кандидат техн. наук, доиент \\ Самарский Государственный Технический Университет \\ 2. Самара \\ Павлова Людмила Викторовна \\ кандидат техн. наук, доцент \\ Самарский Государственный Технический Университет \\ 2. Самара \\ Димеев Артём Маратович \\ магистрант \\ Самарский Государственный Технический Университет \\ 2. Самара
}

\section{RESTORATION OF HIGHWAYS AFTER REPAIR OF COMMUNICATIONS}

Dormidontova Tat'yana Vladimirovna the candidate tehn. Sciences, associate Professor Samara State Technical University Samara

Lyudmila Pavlova candidate tech. Sciences, associate Professor Samara State Technical University Samara

Dimaev Artem Maratovich undergraduate Samara State Technical University Samara

\section{Аннотация}

DOI: $\underline{10.31618 / \text { nas.2413-5291.2020.1.56.226 }}$

Проводимые исследования сводились к изучению технической реализации ремонта коммуникаций, первостепенное внимание уделялось рациональному соотношению между ее стоимостью и техническими характеристиками. Анализ существующих проблем в обозначенной области позволил сформулировать цель работы, которая состоит в исследовании параметров линейных очистных сооружений, влияющих на качество автомобильных дорог.

\section{Abstract}

The conducted research was limited to the study of the technical implementation of repair of communications, priority attention was paid to the rational relationship between its cost and technical 